\title{
Modeling formalin fixation and antigen retrieval with bovine pancreatic RNase A II. Interrelationship of cross-linking, immunoreactivity, and heat treatment
}

\author{
Vladimir K Rait, Lixin Xu, Timothy J O’Leary and Jeffrey T Mason \\ Division of Biophysics, Department of Cellular Pathology and Genetics, Armed Forces Institute of Pathology, \\ Rockville, MD, USA
}

\begin{abstract}
In this study, gel electrophoresis and capture enzyme-linked immunosorbent assay were used to assess the effect of formaldehyde treatment on the structural and immunological properties of bovine pancreatic ribonuclease A (RNase A). Prolonged incubation of RNase A in a 10\% formalin solution leads to the formation of extensive intra- and intermolecular cross-links. However, these formaldehyde cross-links do not completely eliminate the recognition of RNase A by a polyclonal antibody. Comparative immunotitration of monomers, dimers, and oligomers greater than pentamers isolated from formalin-treated RNase A demonstrated that reduction of immunoreactivity due to intramolecular modifications prevails over the excluded volume effect of intermolecular cross-links. The latter only becomes important for intermolecular cross-links involving four or more molecules. The restoration of RNase A immunoreactivity during heating correlates with the reversal of formaldehyde cross-links if the incubation temperature does not exceed the denaturation temperature of the formalin-treated RNase A preparation. We conclude that formaldehyde cross-links stabilize antigens against the denaturing effects of high temperature, but the reversal of these cross-links is necessary for the restoration of immunoreactivity.
\end{abstract}

Laboratory Investigation (2004) 84, 300-306, advance online publication, 26 January 2004; doi:10.1038/labinvest.3700041

Keywords: antigen retrieval; immunohistochemistry; immunoreactivity; formalin fixation; ribonuclease A; enzymelinked immunosorbent assay

In 1991, Shi et al ${ }^{1}$ published their seminal observation that high-temperature incubation of formalinfixed paraffin-embedded tissue sections in buffers for short periods leads to improved immunohistochemical staining. However, more than a decade later, high-temperature antigen retrieval (AR) remains an empirical procedure with several critical parameters that require trial and error optimization. ${ }^{2,3}$ Further improvements in AR will require an in-depth understanding of the chemistry of formaldehyde fixation and the molecular mechanism(s) underlying the AR method.

It is commonly assumed that decreased immunoreactivity in fixed tissues results from formalde-

Correspondence: TJ O’Leary, Department of Cellular Pathology and Genetics, Armed Forces Institute of Pathology, Room 1057D, 1413 Research Boulevard, Building \#101, Rockville, MD 20850, USA.

E-mail: oleary@afip.osd.mil

Received 22 October 2003; accepted 05 November 2003; published online 26 January 2004 hyde-induced cross-linking. However, cross-linking can affect protein immunoreactivity on many levels. The extremely high concentration of proteins and other solutes within tissues ${ }^{4,5}$ leads to the formation of a dense irregular network of cross-links (gelation) that can prevent antibody penetration to the location of its antigen within the tissue. ${ }^{6}$ Even if this primary barrier is partially destroyed by enzymatic etching or thermal reversal of cross-linking, additional effects of formaldehyde can impede antigen-antibody interactions. Antibody binding can be inhibited by steric hindrance (excluded volume effect) arising from the shielding of epitopes on the target antigen due to the close proximity of adjacent molecules to which the antigen is cross-linked. Immunoreactivity can be further compromised by formalin-induced chemical modifications of the amino acid residues within the epitope and by alterations in protein secondary or tertiary structure.

In this study, we used bovine pancreatic ribonuclease A (RNase A) as a model antigen to study the effects of formaldehyde fixation on immuno- 
reactivity. Our results show that prolonged incubation in $10 \%$ formalin reduces, but does not abolish, the binding of polyclonal antibodies to RNase A. We further show that intramolecular modifications by formaldehyde (cross-links and formaldehyde adducts) are more deleterious to antibody binding than the intermolecular cross-linking of small numbers of protein molecules (excluded volume effect). We demonstrate that cross-links play an important role in AR by stabilizing the antigen against thermal denaturation. Finally, we present direct evidence that the reversal of cross-linking is correlated with the restoration of immunoreactivity in a formalin-treated antigen.

\section{Materials and methods}

Bovine pancreatic RNase A, type III-A, was purchased from Sigma Chemical Company (St Louis, MO, USA). Aqueous $37 \%$ formaldehyde (catalog no. BP531) was purchased from Fisher Scientific (Pittsburgh, PA, USA). Two different formulations of formaldehyde-treated RNase A oligomers were prepared. One preparation was enriched in RNase oligomers consisting of five or more intermolecular cross-linked proteins (10\% formalin oligomers), and the other was enriched in monomer and RNase A oligomers consisting of fewer than five intermolecular cross-linked proteins ( $5 \%$ formalin oligomers). The $10 \%$ formalin cross-linked RNase A oligomers were prepared by incubation of the enzyme $(6.5 \mathrm{mg} /$ $\mathrm{ml}$ ) for 9 days in $10 \%$ formaldehyde, $75 \mathrm{mM}$ potassium phosphate buffer ( $\mathrm{pH} 7.4)$ at $23 \pm 1^{\circ} \mathrm{C}$ in a light-protected microtest vial. To obtain the $5 \%$ formalin cross-linked RNase A oligomers, the concentrations of the enzyme and formaldehyde were reduced to $1 \mathrm{mg} / \mathrm{ml}$ and $5 \%$, respectively, and the incubation time was decreased to 1 day at $23 \pm 1^{\circ} \mathrm{C}$. Following incubation, protein samples were freed of formaldehyde by extensive dialysis against phosphate buffer using Slide-A-Lyzer dialysis cassettes with a molecular weight cut-off of $3.5 \mathrm{kDa}$ (Pierce, Rockford, IL, USA). Individual cross-linked RNase A oligomers were isolated from the dialyzed reaction mixtures by gel filtration on prep grade Superdex 75 (Pharmacia Biotech, Uppsala, Sweden) as described by Rait et al. ${ }^{7}$

Native and formaldehyde-treated RNase A concentrations were determined spectrophotometrically assuming that $E^{1 \%}=6.9$ at $280 \mathrm{~nm}^{8}{ }^{8}$ The composition of all the preparations described above was characterized by electrophoresis of dithiothreitol-treated samples in the presence of $0.1 \%$ SDS. Sodium dodecyl sulfate-polyacrylamide gel electrophoresis (SDS-PAGE) was performed on precast NuPAGE Bis-Tris gradient (4-12\%) polyacrylamide gels $(1 \times 80 \times 80 \mathrm{~mm})$ using 2 -( $N$-morpholino $)$ ethanesulfonic acid-SDS running buffer at $\mathrm{pH} 7.3$ (Invitrogen, Carlsbad, CA, USA). Molecular mass standards $(2.5-200 \mathrm{kDa})$ and the Coomassie blue- based colloidal staining kit were also obtained from Invitrogen. Gel images were documented using a Scanjet 5470c (Hewlett-Packard Co., Palo Alto, CA, USA).

The reversal of formaldehyde-induced modifications was performed at a protein concentration of $0.1 \mathrm{mg} / \mathrm{ml}$ in Tris acetate/EDTA (TAE) buffer ( $40 \mathrm{mM}$ Tris, $1 \mathrm{mM}$ EDTA, adjusted to $\mathrm{pH} 4$ with glacial acetic acid).

Polystyrene 96-well Corning microtiter plates (Corning, NY, USA) were used for the enzymelinked immunosorbent assay (ELISA) studies. For antigen detection in direct or capture assays, rabbit anti-RNase A:horseradish peroxide (HRP)-conjugated antibody (RDI-RIBONAabr-HRP) from Research Diagnostics (Flanders, NJ, USA) was used. For capture assays, purified rabbit polyclonal antibody to bovine pancreas RNase A (antibody ab66111) from Abcam (Cambridge, UK) was used as the capture antibody. Coating solution $(10 \mathrm{mM}$ phosphate-buffered saline, PBS), washing solution ( $2 \mathrm{mM}$ imidazol-buffered saline with $0.02 \%$ Tween 20 ), and bovine serum albumin diluent and blocking solutions were all prepared from concentrates supplied by Kirkegaard \& Perry Labs (Gaithersburg, MD, USA). All samples were analyzed in duplicate or triplicate.

For direct ELISA, microtiter wells were coated at $37^{\circ} \mathrm{C}$ for $1 \mathrm{~h}$ with various amounts of antigen in $100 \mu \mathrm{l}$ of PBS. For capture ELISA, coating was performed with the ab6611-1 antibody at a concentration of $1 \mu \mathrm{g} / \mathrm{ml}$ at $37^{\circ} \mathrm{C}$ for $1 \mathrm{~h}$ or at $4^{\circ} \mathrm{C}$ overnight. Capture of antigen and subsequent adsorption of the 1:4000 diluted HRP-conjugated antibody were each carried out at ambient temperature for $1 \mathrm{~h}$. A model 1250 Immunowash microplate washer (Bio-Rad, Hercules, CA, USA) was used to automate well washing at the relevant procedural steps. The HRP detection reaction was initiated by addition of the 2,2'-azino-di-(3-ethylbenzthiazoline-6-sulfonate) substrate and an $\mathrm{H}_{2} \mathrm{O}_{2}$ mixture (ABTS microwell peroxidase substrate system; Kirkegaard \& Perry Labs). Absorbance was monitored at $405 \mathrm{~nm}$ with a model 3550 UV microplate reader (Bio-Rad). The midpoints $\left(\mathrm{OD}_{50}\right)$ of the titration curves and the final (plateau) $405 \mathrm{~nm}$ absorbance values were derived from the absorbance data with the sigmoidal fit tool of Origin software, version 7 (OriginLab Corporation, Northampton, MA, USA).

\section{Results and discussion}

Our objectives in undertaking this work were to answer the following questions: (i) what is the mechanism by which formalin fixation alters an antigen's immunoreactivity, (ii) does the high-temperature restoration of immunoreactivity correlate with the reversal of formaldehyde cross-linking, and (iii) what is the factor that determines the upper temperature limit for maximal restoration of 
immunoreactivity for a particular antigen? To answer these questions, we used a combination of SDS-PAGE and ELISA to monitor cross-linking and immunoreactivity, respectively, of a model antigenRNAse A.

Initially, we compared the ability of direct and capture ELISA to discriminate between native RNase A and RNase A that was incubated at a concentration of $4 \mathrm{mg} / \mathrm{ml}$ in $10 \%$ neutral phosphatebuffered formalin for 1 day and then freed of formaldehyde by dialysis against phosphate buffer (formalin-treated RNase A). As revealed by SDSPAGE (Figure 1, lane 1), the formalin-treated RNase A preparation consisted of a broad set of products, from modified monomer (the lowest band) to an oligomer with 15 or more cross-linked proteins (the top of the upper, unresolved band in lane 1). Lanes 2-6 in Figure 1 characterize selected fractions that were isolated from the preparation by gel chromatography $^{7}$ and subsequently used for ELISA. As shown by SDS-PAGE, formalin-treated monomer (lane 6) has a slightly higher mobility than native RNase A (lane 7). This presumably arises from intramolecular cross-linking. ${ }^{9}$

For direct ELISA, coating of the microtiter plate wells was performed with RNase A dissolved in PBS. The antigen, immobilized via hydrophobic interaction with the polystyrene surface, was then incubated with a rabbit anti-RNase A:HRP-conjugated antibody. The resulting titration curves, shown in Figure 2, have points of inflection (or $\mathrm{OD}_{50}$ values) at $348 \mathrm{ng} / \mathrm{ml}$ for native RNase A (curve 1) and $361 \mathrm{ng} / \mathrm{ml}$ for formalin-treated RNase A (curve 2).

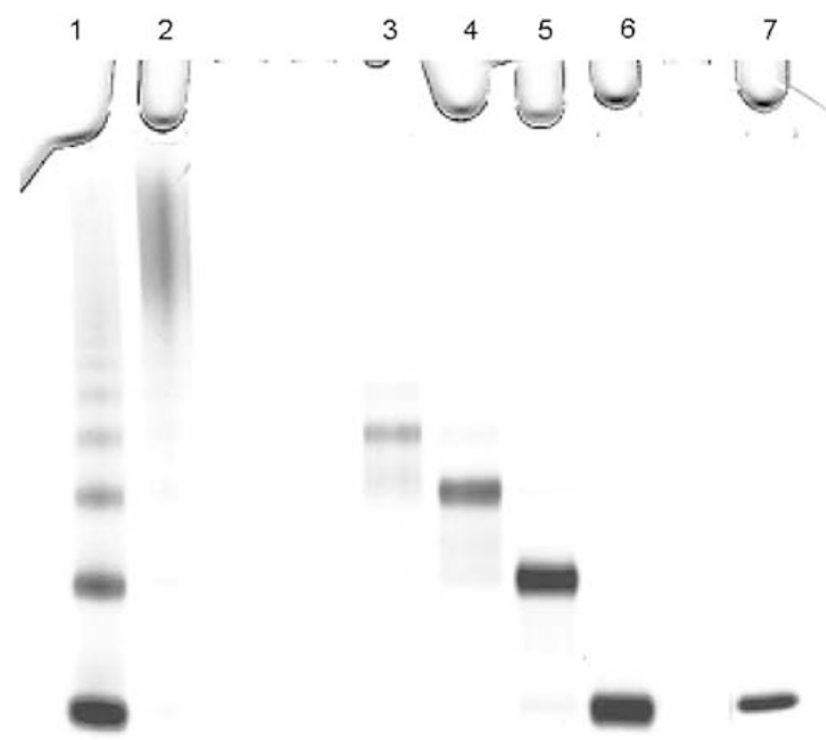

Figure 1 SDS-PAGE of crude formalin-treated RNase A (lane 1), individual fractions of formalin-treated RNase A isolated by gel filtration (lanes: 6, monomer; 5 , dimer; 4 , trimer; 3 , tetramer; 2 , a mixture of oligomers higher than pentamer), and native RNase A (lane 7).
Capture ELISA, in which the antigens were immobilized through interaction with a preadsorbed primary antibody, proved to be more sensitive than direct ELISA, both in the detection of the antigens and in revealing the differences in their binding to antibodies. Curves $1-3$ in Figure 3 correspond to the titration of native RNase $\mathrm{A} \quad\left(\mathrm{OD}_{50}=\right.$ $2.7 \mathrm{ng} / \mathrm{ml}$ ), formalin-treated unfractionated RNase A $\left(\mathrm{OD}_{50}=12.2 \mathrm{ng} / \mathrm{ml}\right)$, and the monomeric fraction of the latter $\left(\mathrm{OD}_{50}=11.5 \mathrm{ng} / \mathrm{ml}\right)$, respectively. The 4.3-fold difference in the $\mathrm{OD}_{50}$ values and the accompanying 1.3-fold difference in the upper plateau values derived from curves 1 and 3 are interpreted as a measure of the disruption to the RNase A immunoreactivity that results from the formation of intramolecular cross-links.

Based on the $\mathrm{OD}_{50}$ values obtained for the titration of cross-linked dimer (9.3 ng/ml, curve 4) and trimer $(11.2 \mathrm{ng} / \mathrm{ml}$, curve 5$)$, the effect of the physically excluded volume due to intermolecular cross-linking is negligible for short oligomers. However, curves $6\left(\mathrm{OD}_{50}=18.3 \mathrm{ng} / \mathrm{ml}\right)$ and $7\left(\mathrm{OD}_{50}=29.6 \mathrm{ng} /\right.$ $\mathrm{ml}$ ), which correspond to tetramer and a mixture of oligomers containing more than five cross-linked molecules, are shifted toward significantly higher concentrations. It should be stressed that these results were obtained with a polyclonal antibody.

Capture ELISA on selected oligomeric fractions of formalin-treated RNase A (see curves 3-7 in Figure 3) also revealed that the plateau values increase with an increase in the number of cross-linked molecules in the fractions. Undoubtedly, this is due to an increasing proportion of bound epitopes per binding site or, in other words, epitope density on the surface. Thus, the nearly identical plateau values for the titration of native RNase A and formalin-treated unfractionated RNase A (curves 1 and 2 in Figure 3)

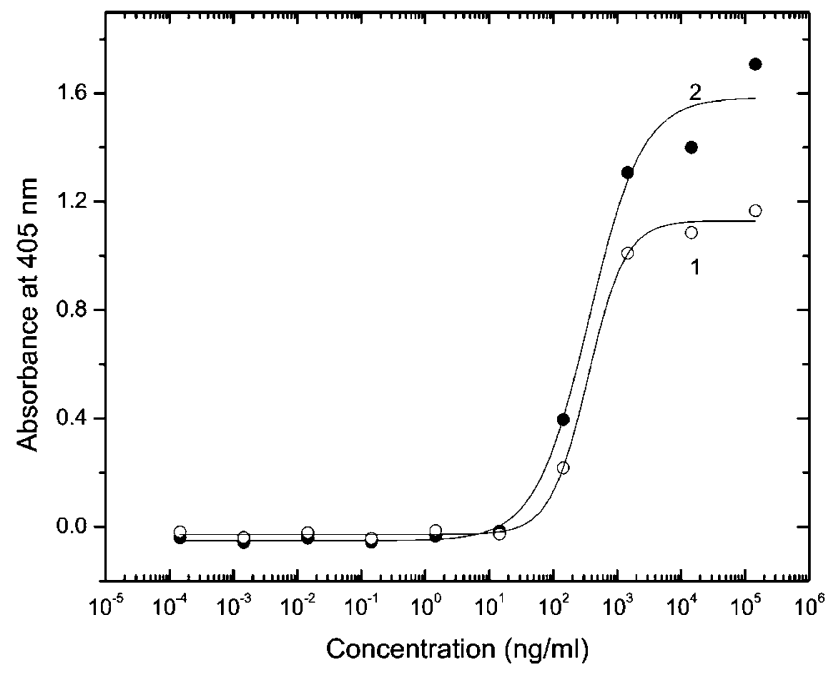

Figure 2 Results of direct ELISA on native RNase A (curve 1) and RNase A incubated in $10 \%$ neutral buffered formalin at a concentration of $4 \mathrm{mg} / \mathrm{ml}$ for 1 day (curve 2). Coating was done at $37^{\circ} \mathrm{C}$ for $1 \mathrm{~h}$. 


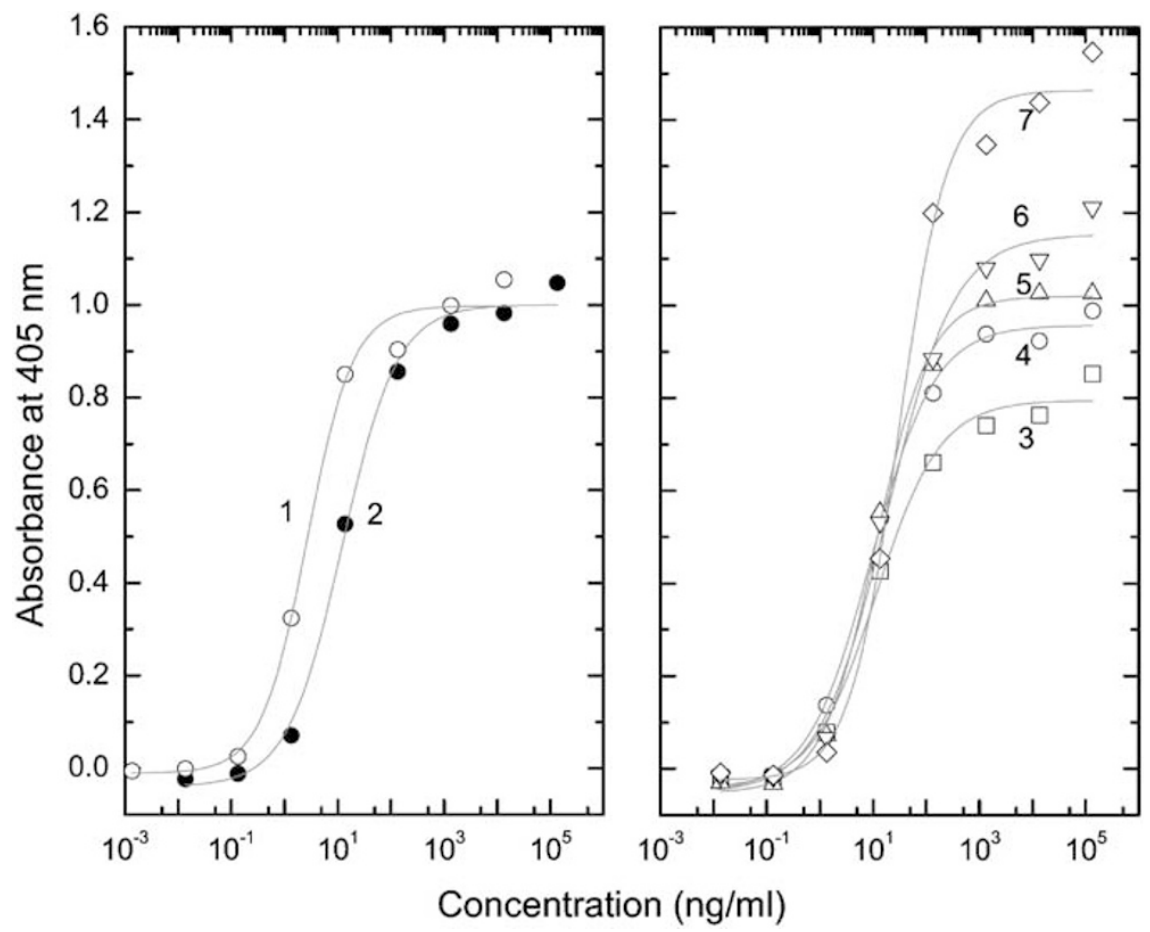

Figure 3 Results of capture ELISA on native RNase A (curve 1), formalin-treated RNase A (curve 2), and individual fractions of formalintreated RNase A (curves: 3, monomer; 4, dimer; 5, trimer; 6, tetramer; and 7, mixture of oligomers higher than pentamer). Coating was done at $37^{\circ} \mathrm{C}$ for $1 \mathrm{~h}$.

are fortuitous, being caused by the particular composition of oligomers present in the formalintreated RNase A preparations that we analyzed.

Comparison of the results of capture ELISA on the native enzyme and the monomeric fraction isolated from crude formalin-treated RNase A unequivocally shows that antibody binding is affected by intramolecular cross-linking, even if the antigen secondary and tertiary structure remain essentially unperturbed as previously reported. ${ }^{7}$ Based on the data in Figure 3 , the disruptive effect of intermolecular cross-linking appears to be less significant than that of intramolecular cross-linking; however, intermolecular cross-linking contributes to a decrease in immunoreactivity when oligomerized RNase A is composed of four or more bound molecules.

Capture ELISA was used to follow the restoration of immunoreactivity in formalin-treated RNase A preparations after incubation at elevated temperatures in TAE buffer at $\mathrm{pH} 4$. A pH of 4 was chosen because previous studies demonstrated this $\mathrm{pH}$ as optimal for the restoration of RNase A enzymatic activity. ${ }^{7}$ Immunoreactivity restoration studies were conducted with both the $10 \%$ formalin oligomers and $5 \%$ formalin oligomers, which were prepared as described in the Materials and methods. As shown in Figure 4, the $10 \%$ formalin oligomers consist mostly of oligomers with seven or more cross-linked proteins (lane 2), whereas the $5 \%$ formalin oligomers have a higher content of modified monomers, dimers, and trimers (lane 4). A 4-h incubation in TAE buffer $(\mathrm{pH} 4)$ at $65^{\circ} \mathrm{C}$ (the denaturation

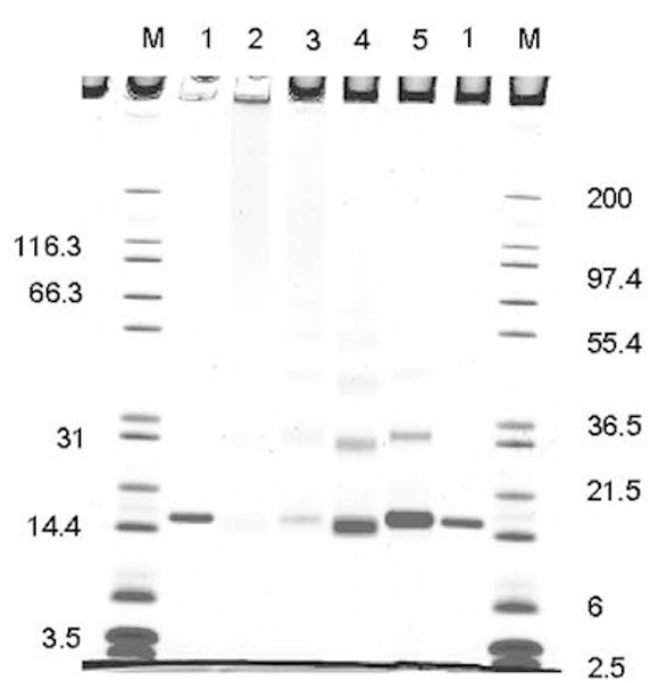

Figure 4 SDS-PAGE of native RNase A (lane 1) and RNase A incubated in $10 \%$ neutral buffered formalin for 9 days (lane 2) or in $5 \%$ neutral buffered formalin for 1 day (lane 4). The latter two samples were then demodified for $4 \mathrm{~h}$ in TAE buffer $(\mathrm{pH} 4)$ at $65^{\circ} \mathrm{C}$ (lane $3,10 \%$ sample; lane $5,5 \%$ sample). M, molecular mass markers in $\mathrm{kDa}$.

temperature $\left(T_{\mathrm{d}}\right)$ of native RNase A) resulted in a significant destruction of intermolecular cross-links in both the $10 \%$ formalin oligomers (lanes 2 vs 3 ) and $5 \%$ formalin oligomers (lanes 4 vs 5 ). The incubation also affected the electrophoretic mobility of each fraction in the samples and restored the 
mobility of modified monomer to that of the untreated enzyme (Figure 4, lane 1).

The structural changes that occurred upon incubation at $65^{\circ} \mathrm{C}$ resulted in the partial restoration of immunoreactivity of the samples as shown in Figure 5 for $5 \%$ formalin oligomers and Figure 6 for $10 \%$ formalin oligomers. In both figures, curve 1 corresponds to unheated native enzyme, curve 2 corresponds to unheated formalin-treated RNase A, and curve 3 corresponds to samples heated at $65^{\circ} \mathrm{C}$ for $4 \mathrm{~h}$ in TAE buffer (pH 4). The restoration of RNase A immunoreactivity upon heating at $65^{\circ} \mathrm{C}$, as shown in Figures 5 and 6 , is clearly correlated with the destruction of formaldehyde cross-links as shown in Figure 4. The data in Figures 4-6 represent the first direct evidence of a relationship between formaldehyde cross-link reversal and immunoreactivity restoration in a formalin-treated antigen resulting from a heat-induced AR procedure. By comparing the results obtained with the 10 and $5 \%$ formalin oligomers, it can be seen that the higher the extent of initial cross-link formation in the RNase A preparation, the less the restoration of RNase immunoreactivity is obtained from a 4-h incubation at $65^{\circ} \mathrm{C}$. This leads to the conclusion that efforts to standardize immunohistochemical staining (see concerns of Shi et $a l^{10}$ ) must begin with standardization of fixation time, which determines the extent of cross-linking. Less obvious, but no less important, is the fact that underfixation can hinder immunohistochemical staining as much as overfixation. ${ }^{11}$ Insufficient fixation in formalin may result in the formation of too few cross-links to stabilize the antigen against the denaturing effects of hightemperature treatment.

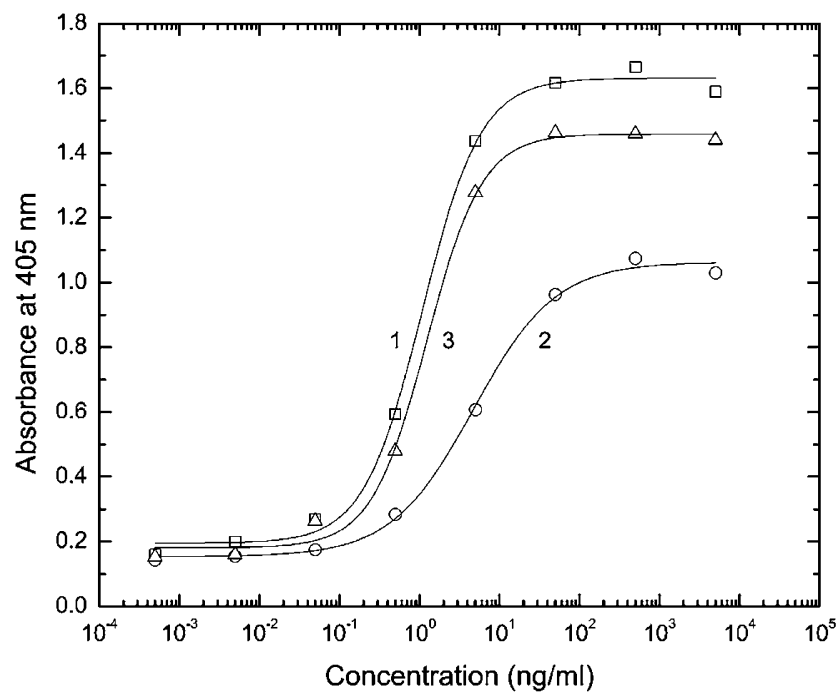

Figure 5 Results of capture ELISA on native RNase A (curve 1) and RNase A incubated in 5\% neutral buffered formalin at a concentration of $1 \mathrm{mg} / \mathrm{ml}$ for 1 day (curve 2) and then demodified for $4 \mathrm{~h}$ in TAE buffer ( $\mathrm{pH} 4$ ) at $65^{\circ} \mathrm{C}$ (curve 3). Coating was done at $4^{\circ} \mathrm{C}$ overnight.
We next examined the structural changes to RNase A 10 and 5\% formalin oligomers that resulted from a 2-h incubation in TAE buffer $(\mathrm{pH} \mathrm{4)}$ at various temperatures (Figure 7). The results for $5 \%$ formalin oligomers are shown in lanes 1-4 for samples before (lane 1) and after incubation in TAE buffer $(\mathrm{pH} 4)$ for $2 \mathrm{~h}$ at $55^{\circ} \mathrm{C}$ (lane 2 ), $75^{\circ} \mathrm{C}$ (lane 3 ), or $95^{\circ} \mathrm{C}$ (lane 4 ). The results for $10 \%$ formalin oligomers are shown in

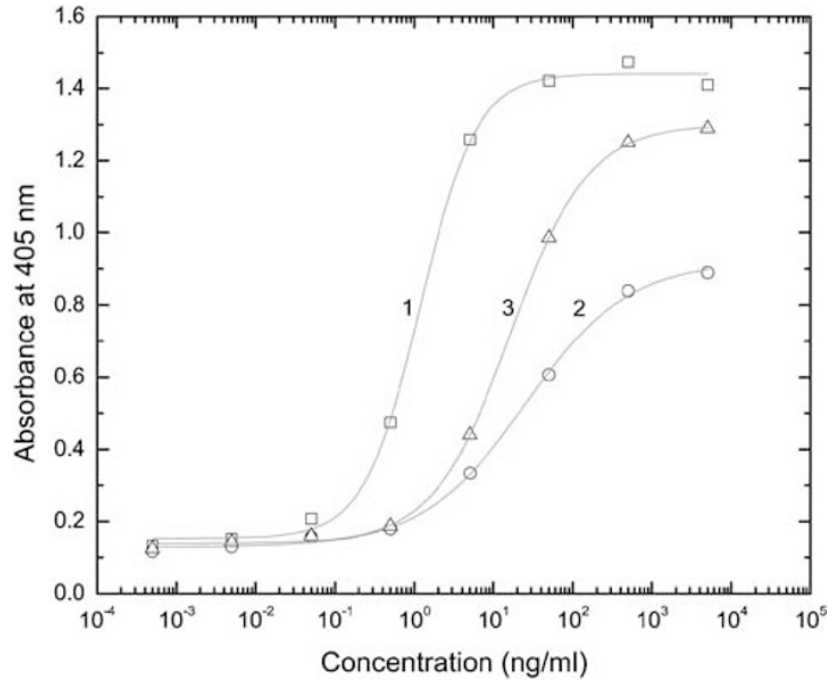

Figure 6 Results of capture ELISA on native RNase A (curve 1) and RNase A $(6.5 \mathrm{mg} / \mathrm{ml})$ incubated in $10 \%$ neutral buffered formalin for 9 days (curve 2) and then demodified for $4 \mathrm{~h}$ in TAE buffer (pH 4 ) at $65^{\circ} \mathrm{C}$ (curve 3). Coating was done at $4^{\circ} \mathrm{C}$ overnight.

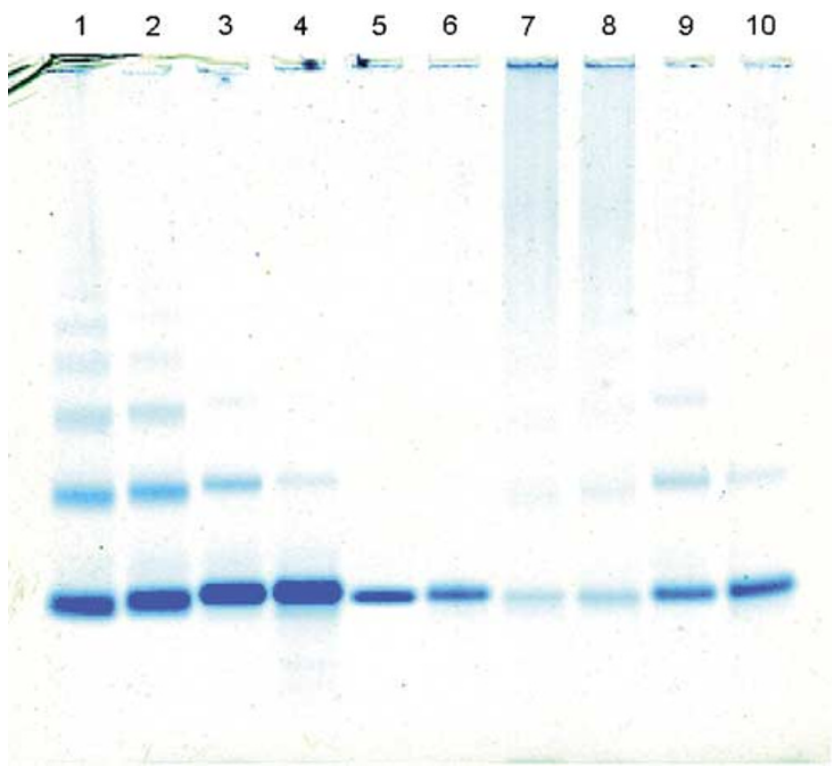

Figure 7 SDS-PAGE of RNase samples. Lanes 1-4: RNase A modified in $5 \%$ neutral buffered formalin for 1 day (lane 1) and then incubated in TAE buffer (pH 4) for $2 \mathrm{~h}$ at $55^{\circ} \mathrm{C}$ (lane 2), $75^{\circ} \mathrm{C}$ (lane 3), or $95^{\circ} \mathrm{C}$ (lane 4). Lanes 5 and 6: unmodified RNase A after incubation in TAE buffer (pH 4) for $2 \mathrm{~h}$ at $55^{\circ} \mathrm{C}$ (lane 5) or $95^{\circ} \mathrm{C}$ (lane 6). Lanes 7-10: RNase A modified in 10\% neutral buffered formalin for 9 days (lane 7) and then incubated in TAE buffer (pH 4) for $2 \mathrm{~h}$ at $55^{\circ} \mathrm{C}$ (lane 8), $75^{\circ} \mathrm{C}$ (lane 9), or $95^{\circ} \mathrm{C}$ (lane 10). 
lanes 7-10 for samples before (lane 7) and after incubation in TAE buffer $(\mathrm{pH} 4)$ for $2 \mathrm{~h}$ at $55^{\circ} \mathrm{C}$ (lane 8 ), $75^{\circ} \mathrm{C}$ (lane 9), or $95^{\circ} \mathrm{C}$ (lane 10). Lanes 5 and 6 are control samples consisting of native RNase A heated for $2 \mathrm{~h}$ in TAE buffer $(\mathrm{pH} 4)$ at $55^{\circ} \mathrm{C}$ (lane 5) or $95^{\circ} \mathrm{C}$ (lane 6). For both formaldehyde-treated RNase A preparations, higher temperatures were more effective in the reversal of formaldehyde cross-links. In fact, the 2 -h incubation at $95^{\circ} \mathrm{C}$ converts both samples into electrophoretically indistinguishable mixtures of monomer and dimer (compare lanes 4 and 10). It is worth noting that the incubation is not accompanied by a noticeable polypeptide chain scission in modified or native RNase A. Products of such a scission would be positioned between the RNase band and the bottom of the gel.

Temperature-induced changes in the immunoreactivity of the formaldehyde-treated samples are not as uniform as the corresponding structural changes revealed by SDS-PAGE. The ELISA data in Figure 8 show a monotonic decrease in immunoreactivity (as measured by the absorbance at $405 \mathrm{~nm}$ ) for the native enzyme (curve 1) over the $55-95^{\circ} \mathrm{C}$ temperature range. In contrast, there is a biphasic dependence on temperature in the case of $5 \%$ (curve 2 ) and $10 \%$ (curve 3 ) formalin oligomers. Also, the heavily cross-linked sample seems to be minimally responsive to the temperature rise from 55 to $65^{\circ} \mathrm{C}$ (curve 3), whereas the signal detected from the sample enriched with modified monomer increases by $20 \%$ (curve 2). We previously determined the $T_{\mathrm{d}}$ to be $65.1^{\circ} \mathrm{C}$ for native RNase $\mathrm{A}, 76.5^{\circ} \mathrm{C}$ for the RNase A $5 \%$ formalin oligomers, and $83.4^{\circ} \mathrm{C}$ for the $10 \%$ formalin oligomers. ${ }^{7}$ From these values, we conclude that, upon exceeding the $T_{\mathrm{d}}$ of the formaldehyde-treated RNase preparation, cross-link

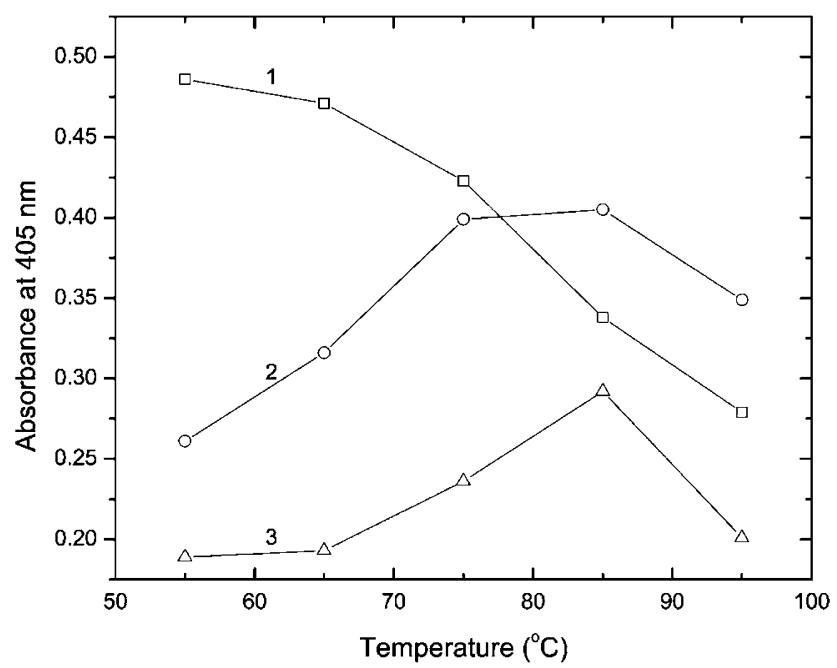

Figure 8 Results of capture ELISA detection of native RNase A (curve 1) and RNase A incubated in 5\% neutral buffered formalin for 1 day (curve 2) or $10 \%$ neutral buffered formalin for 9 days (curve 3), after a 2-h incubation in TAE buffer ( $\mathrm{pH} 4$ ) at different temperatures. Coating was done at $4^{\circ} \mathrm{C}$ overnight. Capturing was done from solutions containing $0.2 \mathrm{ng}$ of antigen in $200 \mu \mathrm{l}$ of PBS. destruction is no longer accompanied by restoration of immunoreactivity. The explanation for this observation is straightforward. Below the $T_{\mathrm{d}}$, the thermal reversal of formaldehyde cross-links yields a thermally stable protein that regains a significant fraction of its immunoreactivity. Above the $T_{\mathrm{d}}$, the restoration of immunoreactivity that results from the thermal reversal of formaldehyde cross-links must compete with the thermal denaturation of the protein preparation, which significantly reduces immunoreactivity. The more the incubation temperature for AR exceeds the $T_{\mathrm{d}}$ of the protein, the more the balance swings in favor of protein denaturation and a loss of immunoreactivity. Given this scenario, we hypothesize that the ideal method for AR is one where the AR incubation temperature is decreased over time to follow the kinetics of crosslink destruction. However, the initial temperature at the start of the AR protocol may need to be even higher than the constant temperatures currently used in some 'superheating' protocols. ${ }^{12}$

How does high temperature promote the reversal of formaldehyde-induced cross-links? In Figure 9, we show the structural diagrams of the amino methylol adduct formed by an Asn residue (a) and an Asn cross-linked with a Lys residue (b). Within each structure, there is the possibility of the formation of a six-atom ring stabilized by a hydrogen bond. In fact, similar structural fragments can also be formed in cross-links between Lys and Gln, Arg, and other amino acids involved in cross-linking. Thus, it is likely that high-temperature reversal of formaldehyde-induced cross-links and methylol

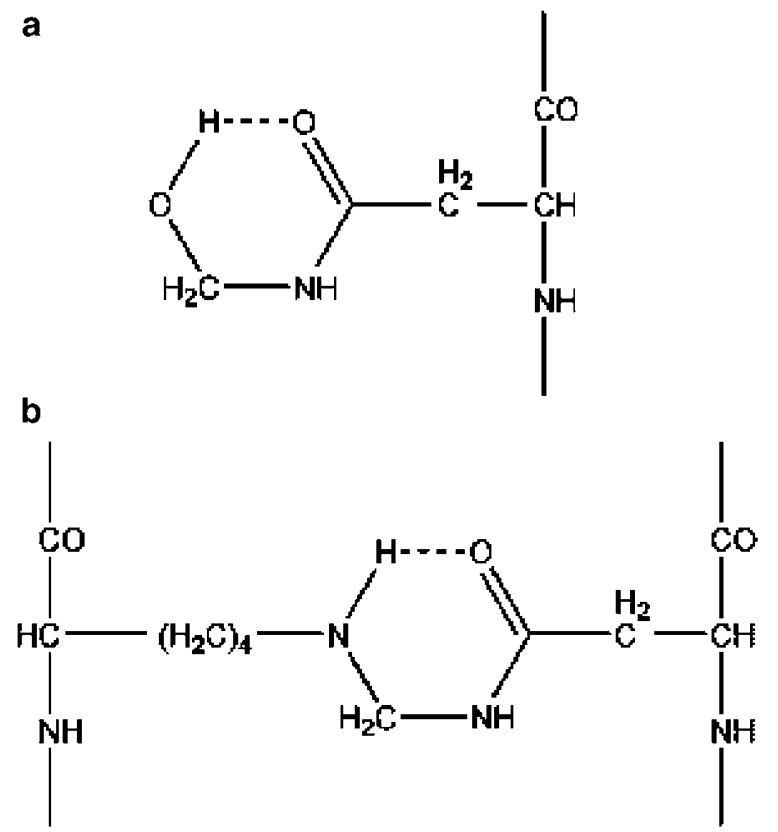

Figure 9 Molecular diagrams of proposed hydrogen bond formation in (a) amino methylol adducts formed by Asn and (b) an Asn cross-linked with a Lys residue. 
adducts is initiated by disruption of this intrinsic hydrogen bond.

In conclusion, we have shown that prolonged incubation in $10 \%$ formalin does not completely abolish recognition of bovine pancreatic RNase A by a polyclonal antibody. The reduction of antigenicity due to intramolecular modifications is shown to be more important than the excluded volume effect, which contributes only for cross-linking of four or more molecules. The restoration of immunoreactivity during heating of formaldehyde-treated RNase A is correlated with the reversal of cross-linking as long as the incubation temperature does not exceed the $T_{\mathrm{d}}$ of the formalin-treated RNase A preparation.

\section{Acknowledgement}

We thank Dr Marilyn J Mason for her excellent editorial assistance in the preparation of this manuscript. This work was supported in part by a grant from the National Cancer Institute (1R21 CA091227-01) and by the American Registry of Pathology.

\section{References}

1 Shi SR, Key ME, Kalra KL. Antigen retrieval in formalin-fixed, paraffin-embedded tissues: an enhancement method for immunohistochemical staining based on microwave oven heating of tissue sections. J Histochem Cytochem 1991;39:741-748.

2 Shi SR, Cote RJ, Yang C, et al. Development of an optimal protocol for antigen retrieval: a 'test battery' approach exemplified with reference to the staining of retinoblastoma protein (pRB) in formalin-fixed paraffin sections. J Pathol 1996;179:347-352.

3 Miller RT, Swanson PE, Wick MR. Fixation and epitope retrieval in diagnostic immunohistochemistry: a concise review with practical considerations. Appl Immunohistochem Mol Morphol 2000;8:228-235.

4 Ellis RJ. Macromolecular crowding: obvious but underappreciated. Trends Biochem Sci 2001;26:597-604.

5 Verkman AS. Solute and macromolecule diffusion in cellular aqueous compartments. Trends Biochem Sci 2002;27:27-33.

6 Hopwood D. Microwaves and heat in aldehyde fixation: model experiments with bovine serum albumin. Methods 1998;15:119-122.

7 Rait VK, O’Leary TJ, Mason JT. Modeling formalin fixation and antigen retrieval with bovine pancreatic RNase A. I. Structural and functional alterations 2004; 84: 292-299.

8 Pace CN, Vajdos F, Fee L, et al. How to measure and predict the molar absorption coefficient of a protein. Protein Sci 1995;4:2411-2423.

9 Paliwal R, London E. Comparison of the conformation, hydrophobicity, and model membrane interactions of diphtheria toxin to those of formaldehyde-treated toxin (diphtheria toxoid): formaldehyde stabilization of the native conformation inhibits changes that allow membrane insertion. Biochemistry 1996;35:2374-2379.

10 Shi SR, Cote RJ, Taylor CR. Antigen retrieval immunohistochemistry: past, present, and future. J Histochem Cytochem 1997;45:327-343.

11 De Marzo AM, Fedor HH, Gage WR, et al. Inadequate formalin fixation decreases reliability of p27 immunohistochemical staining: probing optimal fixation time using high-density tissue microarrays. Hum Pathol 2002;33:756-760.

12 Leong AS, Lee ES, Yin H, et al. Superheating antigen retrieval. Appl Immunohistochem Mol Morphol 2002;10:263-268. 